\title{
A cometary perspective of Enceladus
}

\author{
Daniel C. Boice and Raymond Goldstein \\ Space Science \& Engineering Div., Southwest Research Institute, \\ 6220 Culebra Road, San Antonio, TX 78238 USA \\ email: dboice@swri.edu \\ email: rgoldstein@swri.edu
}

\begin{abstract}
Icy plumes venting from Enceladus draw obvious comparisons to such features seen in comets. This paper outlines a consistent evolution from cometary activity to larger icy bodies in the outer solar system. The major differences are due to the systematic effects of increased gravity, including more spherical solid bodies (self-gravity), less porosity, the possible existence of liquid water due to internal sources of heat (Enceladus) versus possible cometary cyrovolcanism, internal inhomogeneities leading to jet-like features, and the possibility of a quasi-bound dusty gas atmosphere, as opposed to the extensive exospheres of comets. Similarities exist also, including gas and dust emission and the filamentary nature of jet-like features (caused by surface topography in comets), surface evolution by dust accumulation, heat and gas transport through the surface layers, among others. Initial results regarding the plume chemistry and comparisons to CAPS ion data show similarities too. Others have considered additional effects such as, charging of particles, micrometeorite impacts and complex interactions with the E-ring neutrals and plasma in great detail so these topics remain outside the scope of this paper.
\end{abstract}

Keywords. Planets and Satellites: Enceladus, Comets: Chiron, Comets: General

\section{Introduction}

The discovery of icy plumes emanating from Saturn's moon Enceladus by the Cassini spacecraft has raised questions about the cometary nature of this small satellite. The release of gas and dust from cometary nuclei is restricted to 'jets' or plumes also and this activity has been observed in comets at distances much further than Saturns orbit, including comet-like activity and a resolved coma of the Centaur Chiron. Enceladus and Chiron have sizes that are much larger than cometary nuclei but their atmospheres are still largely unbound, similar to the exospheres of comets. With Chiron, Enceladus may represent a transitional object in this respect, intermediate to the tightly bound, thin atmospheres typical of planets and large satellites and the greatly extended atmospheres in free expansion typical of cometary comae.

Measurements of the neutral and ion composition of the plumes reveal the presence of water group species, nitrogen-bearing molecules, and other species that have been found in comets (see Table 1). The nature of the volatile materials in Enceladus may also bear similarities with ideas of cometary ices (see Table 2). In other respects, the large size of Enceladus relative to comets and the presence of Saturn and its magnetosphere nearby, brings into question the validity of applying scaling laws to cometary results in order to understand the environment surrounding Enceladus. In addition, release mechanisms for the icy grains and gases at Enceladus, including liquid water mixtures below the cold, icy surface, are not thought to be applicable to comets. These issues and others are discussed as we offer a cometary perspective on our current understanding of Enceladus. 
Table 1. Volatile composition of Enceladus and a typical comet.

\begin{tabular}{ccccc}
\hline Body & $\mathbf{H}_{2} \mathbf{0}$ & $\mathbf{C H}_{4}$ & $\mathbf{C O} / \mathbf{N}_{2}$ & $\mathbf{C O}_{2}$ \\
\hline Enceladus & 100 & 1.9 & 4.4 & 3.5 \\
Comets & 100 & $0.14-1.5$ & $3.5-14$ & $3-6$ \\
\hline
\end{tabular}

Table 2. Molecular production rates for ices at Enceladus (near perihelion ${ }^{1}$ ).

\begin{tabular}{|c|c|c|c|c|}
\hline Parameter & $\mathrm{CO}$ & $\mathrm{CO}_{2}$ & $\begin{array}{c}\mathrm{H}_{2} \mathrm{O} \\
\text { (Crystalline) }\end{array}$ & $\begin{array}{c}\mathrm{H}_{2} \mathrm{O} \\
\text { (Amorphous) }\end{array}$ \\
\hline $\begin{array}{c}\mathrm{Z}_{\text {surface }} \\
\text { [molecules } / \mathrm{cm}^{2} \mathrm{~s} \text { ] }\end{array}$ & $10^{17}$ & $2 \cdot 10^{16}$ & $10^{10}$ & $10^{16}$ \\
\hline $\begin{array}{c}\mathrm{n}_{\text {surface }} \\
{\left[/ \mathrm{cm}^{3}\right]}\end{array}$ & $8.3 \cdot 10^{12}$ & $1.3 \cdot 10^{12}$ & $3.5 \cdot 10^{5}$ & $3.1 \cdot 10^{11}$ \\
\hline $\begin{array}{c}\mathrm{T}_{\text {surface }} \\
{[\mathrm{K}]}\end{array}$ & 35 & 94 & 105 & 115 \\
\hline $\begin{array}{l}\mathrm{v}_{\text {surface }} \\
{[\mathrm{km} / \mathrm{s}]}\end{array}$ & $0.12^{2}$ & $0.16^{2}$ & $0.21^{2}$ & 0.35 \\
\hline
\end{tabular}

Notes:

${ }^{1} \mathrm{r}_{h}=9.0 \mathrm{AU}$.

${ }^{2}$ Average speed is less than the escape velocity of Enceladus $(0.24 \mathrm{~km} / \mathrm{s})$.

\section{Observations and in situ Measurements}

The Cassini spacecraft is opening a new chapter in our understanding of the icy moon Enceladus and the role of venting as the major source of neutral gasses and dust in its surrounding environment. Recent Cassini observations have established that the icy moon Enceladus is actively venting and ejecting water, other neutral molecules, and dust; indicating that it is the origin of the surrounding atmosphere and E-ring torus. The interactions between the subsurface gas source and the jet-like activity of the neutrals and dust are critical processes with significant implications for the evolution of the broader environment. Understanding these interactions would enable us to establish source properties during past and future Cassini flybys and investigate its time variability from encounter to encounter. Due to many similarities to cometary behavior, comparisons to cometary models may allow us to make better estimates of the gas and dust production rates at Enceladus and the likely composition of neutrals and ions in the venting region.

\section{Enceladus and the Surrounding Environment}

The Cassini spacecraft has performed several close flybys of Enceladus, revealing the moon's surface and environment in great detail and discovering a water-rich plume venting from its South Polar Region (SPR). The composition of the Enceladean plume as measured by the INMS instrument is similar to that seen at most comets (Table 1), containing mostly water vapor as well as minor components of $\mathrm{CO}$ (and possibly $\mathrm{N}_{2}$ ), $\mathrm{CH}_{4}, \mathrm{CO}_{2}$, and simple and complex hydrocarbons, such as propane, ethane, and acetylene (Waite et al. 2006). This discovery, along with the presence of escaping internal heat and very few impact craters in the SPR, indicates that Enceladus is geologically active. The discovery of the plume supports the notion that material released from Enceladus is the source of the E-ring, composed of water ice grains that are primarily 0.3 to 3 $\mathrm{mm}$ in size (Nicholson et al. 1996). There are two mechanisms contributing to the ring 
(Spahn et al. 2006). The most important source of particles comes from the cryovolcanic plume. For gas or dust to escape from a small icy body, the radial component of the gas velocity must exceed the gravitational attraction. For a satellite orbiting a planet we must also consider the Hill sphere. For Enceladus, the Hill sphere radius is $949 \mathrm{~km}$, the escape velocity from the surface is $239 \mathrm{~m} / \mathrm{s}$ and this speed is reduced to $205 \mathrm{~m} / \mathrm{s}$ at the Hill sphere radius. While a majority of particles fall back to the surface, some escape and enter orbit around Saturn. The second mechanism comes from hypervelocity micrometeoroid impacts of Enceladus, raising dust particles from the surface, but this alone cannot explain the dust data (Spahn et al. 2006). This leaves gas entrainment of dust from the vents as a viable source. Dusty gas interaction is necessary to understand the complex interactions in the plumes and its surrounding environment. We estimate that the maximum grain size that can be lifted due to gas entrainment is about 10 $100 \mu \mathrm{m}$. This doesn't appear to be sufficient to lift the icy grains seen in the E-ring.

Cassini images show fine structures within the plumes, revealing numerous filaments (perhaps due to numerous distinct vents) within a larger, faint component extending out nearly $500 \mathrm{~km}$ from the surface. Cassini CDA data are compatible with a dust source and the UVIS later observed gas jets coinciding with the dust jet-like features during recent flybys of Enceladus. Various investigations have estimated $\mathrm{H}_{2} \mathrm{O}$ release rates necessary to maintain a steady state of about $2 \cdot 10^{27} \mathrm{H}_{2} \mathrm{O}$ molecules/s (Shemansky et al. 1993), possibly as high as $3.75 \cdot 10^{27}$ (Jurac et al. 2002) or $10^{28} \mathrm{H}_{2} \mathrm{O}$ molecules/s (Jurac \& Richardson 2005). Potential $\mathrm{H}_{2} \mathrm{O}$ sources identified by these investigators include sputtering and collisions; however, the rates of these processes are not sufficient to replace the lost neutrals (Jurac et al. 2002). Plume modeling near closest approach gives the total $\mathrm{H}_{2} \mathrm{O}$ production rate from Enceladus of 1.5 to $4.5 \cdot 10^{26}$ molecules/s, and the total gas production rate 1.7 to $5.0 \cdot 10^{26}$ molecules/s, assuming the gas is $90 \% \mathrm{H}_{2} \mathrm{O}$ from INMS measurements. The bounds of the minimum and maximum estimates indicate a highly variable source rate with time scales of less than 1 hour over the range from $10^{26}$ to $3 \cdot 10^{27}$ molecules/s. The fissures from which the gas originates must be large enough to allow semicollisional gas flow below the surface (i.e., the size of the fissure must be larger than the mean free path between molecules). If the fissures are too small, molecules will stick to the cold surfaces, eventually sealing it off. Even the average temperature observed by the CIRS of $140 \mathrm{~K}$ is far below the sublimation temperature so most molecules striking the surface would stick (Waite et al. 2006).

\section{Source Mechanisms}

Analysis of the outgassing suggests that it originates from a body of sub-surface liquid water, which along with the unique chemistry found in the plume has important astrobiology implications. Moons of gas giants can become trapped in orbital resonances that lead to forced libration or orbital eccentricity; proximity to the planet can then lead to tidal heating of the satellite's interior, offering a possible explanation for the activity. However, recent work has shown that Enceladus doesn't oscillate about the tidal equilibrium (Meyer \& Wisdom 2008) as required by the tidal heating model of Ojakangas \& Stevenson (1986), so other mechanisms must be responsible. The combined analysis of imaging, mass spectrometry, and magnetospheric data suggests that the observed south polar plume emanates from pressurized sub-surface chambers, similar to geysers on Earth (Porco et al. 2006). Since no ammonia was found in the vented material, which could act as an anti-freeze, the heated, pressurized chamber would consist of nearly pure liquid water with a temperature of at least $270 \mathrm{~K}$. Pure water would require more energy to melt, either from tidal or radiogenic sources, than an ammonia-water mixture. Another 
possible method for generating a plume is sublimation of warm surface ice. Temperatures found near the south pole by CIRS range from $85-90 \mathrm{~K}$ to as high as $157 \mathrm{~K}$ in small areas. This is too warm to be explained by solar heating, indicating heating from the interior of Enceladus (Spencer et al. 2006). Ice at these temperatures is warm enough to sublimate at a much faster rate than the background surface, thus generating a plume. This hypothesis is attractive since the sub-surface layer heating the surface water ice could be a mixture of ammonia and water at temperatures as low as $170 \mathrm{~K}$, and thus not as much energy is required to produce the plume activity. However, the abundance of particles in the south polar plume favors the cold geyser model, as opposed to ice sublimation (Porco et al. 2006). Alternatively, Kieffer et al. (2006) suggest that the plumes originate from clathrate hydrates; where carbon dioxide, methane, and nitrogen are released when exposed to the vacuum of space by the active, tiger stripe fractures. This hypothesis would not require the amount of heat needed to melt water ice as required by the cold geyser model, and would explain the lack of ammonia. An additional possibility is the presence of amorphous water ice below the Enceladean surface. The exothermic phase change from amorphous to crystalline ice occurs in the temperature regime at Enceladus and releases sufficient water gas to explain the observed release rate as our preliminary modeling indicates (see Table 2). These mechanisms are appropriate for comets when gravity and low porosity considerations are included.

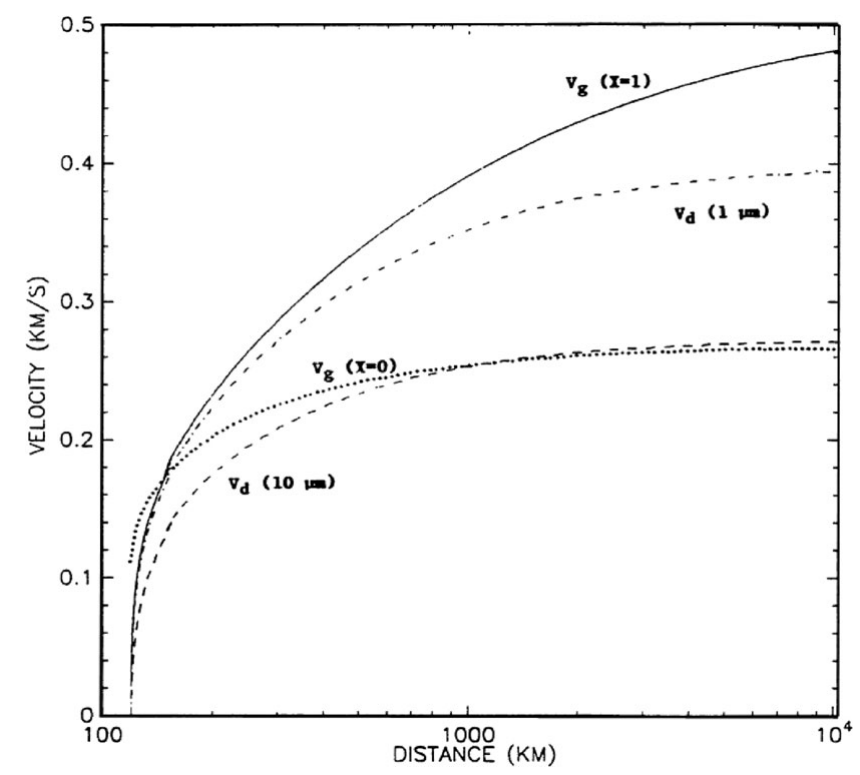

Figure 1. Gas and dust speeds for Chiron at perihelion. The dust acceleration region is within $\sim 10 \mathrm{R}_{\text {Chiron }}$. For comparison, the dotted line is the gas speed for a dust-free model $(\chi=0)$.

\section{The Dusty Gas Outflow}

To test the cometary approach for Enceladus, a preliminary model of Chiron that treats the physics and chemistry of the comet coma in great detail (Schmidt et al. 1988) was prepared by Boice et al. (1991) and Boice \& Huebner (1993). CO was assumed to be the only volatile. Being a diatomic molecule, $\mathrm{CO}$ is not an efficient emitter in the infrared so the radiative cooling term is negligible. In the simulations, gas and dust are rapidly accelerated upon leaving the nucleus as illustrated in Figure 1 ( $\chi$ is the dust-to-gas mass 
ratio). For standard dust densities, small particles are more efficiently entrained with the gas flow than large particles, resulting in higher terminal speeds. The acceleration zone for all particles is approximately within 10 radii of the surface.

The inclusion of dust has two important effects on the gas flow. The first is an initial mass-loading of the gas, maintaining the gas velocity at subsonic values close to the surface of the nucleus. The second effect is a strong thermal coupling of the gas and dust near the nucleus as shown in Figure 2. Upon release, the dust heats rapidly to its radiative equilibrium value of $95 \mathrm{~K}$. Collisions of molecules with dust particles heat the $\mathrm{CO}$ gas (initially at $30 \mathrm{~K}$ ) to $85 \mathrm{~K}$ within a Chiron radius. This results in a terminal gas velocity about $80 \%$ higher than that calculated from a pure gas model. Even with a modest amount of dust (dust-to-gas mass ratio of 0.1 ), the gas is significantly heated in the near-nucleus region. Recent progress of the model includes the addition of the gravity to Chiron and the incorporation of dust fragmentation and distributed coma sources of gas-phase species related to the dust.

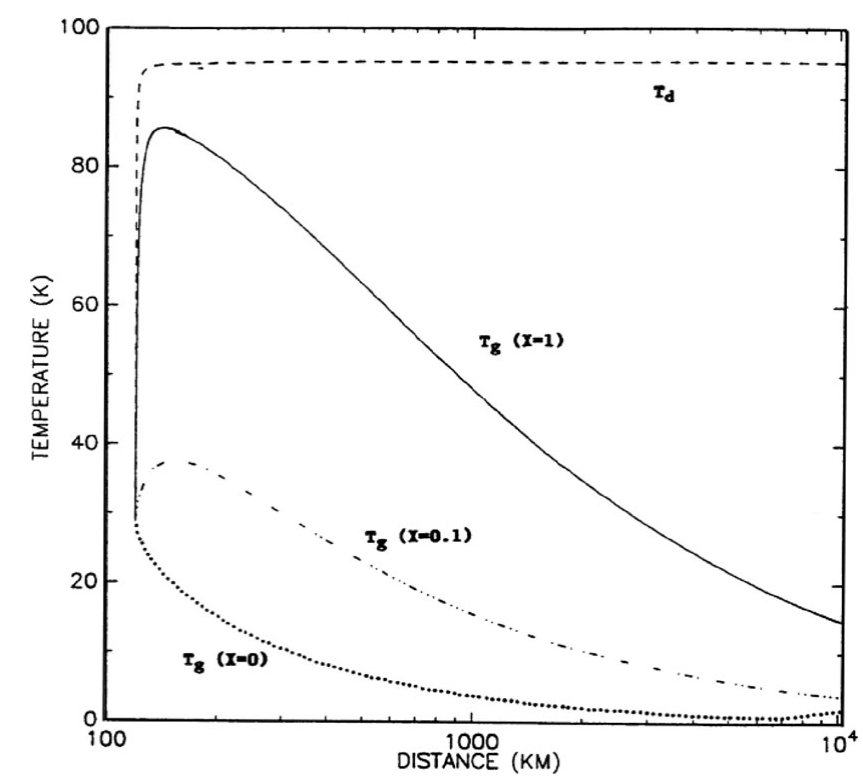

Figure 2. Temperature profiles of the gas and dust for Chiron at perihelion. Upon release from the nucleus, both sizes of dust quickly reach radiative equilibrium at $95 \mathrm{~K}$. The gas is strongly heated by the dust in the near-nucleus region for the $\chi=1$ case. Even with a modest dust-to-gas mass ratio $(\chi=0.1)$, the gas is heated significantly above the dust-free model $(\chi=0)$.

\section{Summary and Conclusion}

This study represents a consistent evolution from our comet nucleus and coma experience to larger icy bodies in the outer solar system. The major difference is the systematic effects of increased gravity, including more spherical solid bodies (self-gravity), less porosity, the possible existence of liquid water due to internal sources of heat (Enceladus) versus possible cometary cyrovolcanism (Belton et al. 2008), internal inhomogeneities leading to jet-like features, and the possibility of a quasi-bound dusty gas atmosphere, as opposed to the extensive exospheres of comets (Johnson et al. 2008). Similarities exist also, including jet-like features with filaments (caused by surface topography in comets) gas and dust emission, surface evolution by dust accumulation, heat and gas transport 
through the surface layers, and others. Initial results regarding the plume chemistry and comparisons to CAPS ion data show similarities too (Boice \& Goldstein 2008, Goldstein et al. 2008). Other effects that need to be considered in a realistic model include charging of particles, micrometeorite impacts and complex interactions with the E-ring neutrals and plasma. These topics remain outside the scope of this work as other researchers have considered them in great detail.

\section{Acknowledgements}

We acknowledge support from the SwRI Internal Research \& Development Program, the NASA Cassini (CAPS) Mission, and the NSF Planetary Astronomy Program.

\section{References}

Belton, M. J. S., Feldman, P. D., A'Hearn, M. F., \& Carcich, B. 2008, Icarus, 198, 189

Boice, D. C. \& Goldstein, R. 2008, Eos Trans. AGU, 89(53), \#P23B-1368

Boice, D. C., Stern, S. A., \& Huebner, W. F. 1991, LPSC XXII, 22, 121

Boice, D. C. \& Huebner, W. F. 1993, in: W. F. Huebner (ed.), Workshop on the Activity of Distant Comets (San Antonio: SwRI), p. 134

Goldstein, R., Boice, D. C., Young, D. T., Reisenfeld, D. B., \& Smith, H. T. 2008, Eos Trans. $A G U, 89(53)$, \#P23B-1382

Johnson, R. E., Combi, M. R., Fox, J. L., Ip, W.-H., Leblanc, F., McGrath, M. A., Shematovich, V. I., Strobel, D. F., \& Waite, J. H. 2008, Space Science Reviews, 139, 355

Jurac, S., McGrath, M. A., Johnson, R. E., Richardson, J. D., Vasyliunas, V. M., \& Eviatar, A. 2002, Geophys. Res. Lett., 29, 25

Jurac, S. \& Richardson, J. D. 2005, J. Geophys. Res., 110, A09220

Kieffer, S. W., Lu, X., Bethke, C. M., Spencer, J. R., Marshak, S., \& Navrotsky, A. 2006, Science, 314,1764

Meyer, J. \& Wisdom, J. 2008, Icarus, 198, 178

Nicholson, P. D., Showalter, M. R., Dones, L., French, R. G., Larson, S. M., Lissauer, J. J., MeGhee, C. A., Seitzer, P., Sicardy, B., \& Danielson, G. E. 1996, Science, 272, 509

Ojakangas, G. W. \& Stevenson, D. J. 1986, Icarus, 66, 341

Porco, C. C., Helfenstein, P., Thomas, P. C., Ingersoll, A. P., Wisdom, J., West, R., Neukum, G., Denk, T., Wagner, R., Roatsch, T., Kieffer, S., Turtle, E., McEwen, A., Johnson, T. V., Rathbun, J., Veverka, J., Wilson, D., Perry, J., Spitale, J., Brahic, A., Burns, J. A., Del Genio, A. D., Dones, L., Murray, C. D., \& Squyres, S. 2006, Science, 311, 1393

Schmidt, H. U., Wegmann, R., Huebner, W. F., \& Boice, D. C. 1988, Comp. Phys. Comm., 49, 17

Shemansky, D. E., Matheson, P., Hall, D. T., Hu, H.-Y., \& Tripp, T. M. 1993, Nature 363, 329

Spahn, U., Schmidt, J, Albers, N., Hörning, M., Makuch, M., Seiß, M., Kempf, S., Srama, R., Dikarev, V., Helfert, S., Moragas-Klostermeyer, G., Krivov, A.V., Sremčević, M., Tuzzolino, A. J., Economou, T., \& Grün, E. 2006, Science, 311, 1416

Spencer, J. R., Pearl, J. C., Segura, M., Flasar, F. M., Mamoutkine, A., Romani, P., Buratti, B. J., Hendrix, A. R., Spilker, L. J., \& Lopes, R. M. C. 2006, Science, 311, 1401

Waite, J. H., Combi, M. R., Ip, W.-H., Cravens, T. E., McNutt, R. L., Kasprzak, W., Yelle, R., Luhmann, J., Niemann, H., Gell, D., Magee, B., Fletcher, G., Lunine, J., \& Tseng, W.-L. 2006, Science, 311, 1419 